\title{
Biomarkers of doping: uses, discovery and validation
}

\author{
Reid Aikin*,1, Norbert Baume ${ }^{1}$, Tristan Equey ${ }^{1}$ \& Olivier Rabin ${ }^{1}$ \\ ${ }^{1}$ World Anti-Doping Agency, 800 Rue du Square-Victoria Suite 1700, Montreal, QC H4Z 1B7, Canada \\ *Author for correspondence: reid.aikin@wada-ama.org
}

\begin{abstract}
A biomarker of doping indicates the biological response to the use of a prohibited substance or method. Uncovering novel biomarkers of doping is a key objective in order to improve antidoping outcomes such as the detection of doping and changing athlete behavior toward doping practices. While the antidoping field has been successful in validating novel metabolites of prohibited substances, there has been less success in developing new biomarkers of doping. Employing the most suitable study designs and analytical approaches is critical to successfully uncovering novel biomarkers of doping with a high potential for translation into routine analysis. Here we argue that the antidoping field is well positioned for biomarker discovery and outline considerations for the development of novel biomarkers of doping.
\end{abstract}

First draft submitted: 17 February 2020; Accepted for publication: 22 April 2020; Published online: 1 June 2020

Keywords: antidoping • athlete biological passport • biomarkers

A key aspect of the fight against doping is the ability to establish the presence or use of prohibited substances in samples provided by athletes with a high degree of certainty. The presence of a prohibited substance is most commonly established by direct detection of the substance or its metabolite(s) in the athlete's biological sample. In addition, the ability of biomarkers of doping to establish the use of a prohibited substance has proven beneficial not only to establish the use of foreign substances, but also for pseudo-endogenous substances that are more difficult to distinguish from endogenously produced compounds with similar physico-chemical properties.

When a biomarker of doping is employed to establish the use by an athlete of a prohibited substance or method (in the absence of the identification of a prohibited substance), a forensic approach is employed to infer use, against the direction of causality, through the application of Bayesian inference (Figure 1). Evidence of doping can be provided by evaluation of biomarker variations over time followed by an evaluation of the relative likelihoods of such variations being due to doping or to other causes. This approach is harmonized in antidoping through the Athlete Biological Passport (ABP) [1]. Such an approach requires a detailed understanding of the sources of variation of the selected biomarker, knowledge of potential confounding factors and harmonized laboratory analysis.

Beyond their use to directly sanction an athlete and arguably with a larger overall positive impact on antidoping outcomes, biomarkers of doping can also drive other antidoping decisions such as the choice to collect additional samples from the athlete, to further analyze existing samples by other analytical methods, to investigate an athlete or group of athletes or to place samples for that athlete into long-term storage for future analysis (Figure 2). Biomarkers of doping can be used to estimate the prevalence of doping [2] and provide an indication of changes in athlete behaviors over time [3]. Athlete testimony suggests that the application of biomarker monitoring through the ABP significantly altered the options and effectiveness of doping strategies available for cheating athletes [4,5]. Therefore, a key target of antidoping science is the continued improvement of the ABP through the development of novel biomarkers of doping.

Despite the significant potential to improve our ability to uncover changes in the condition of an individual, biomarker discovery and validation remain difficult across all fields and key challenges and strategic considerations for biomarker development have been reviewed elsewhere (for example [6-10]). Here, we outline some unique characteristics of biomarkers used for antidoping purposes, particularly those used within the framework of the ABP. We propose that antidoping is well positioned for successful biomarker development and outline specific 


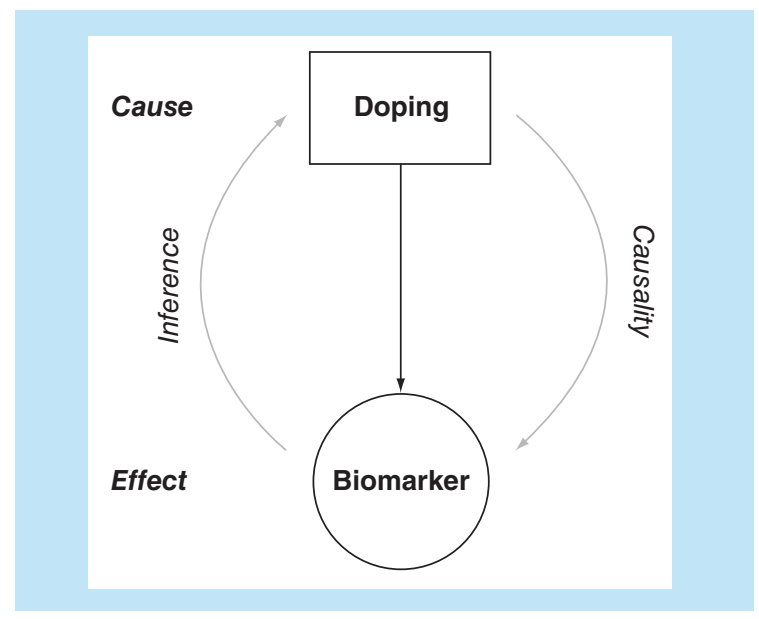

Figure 1. Causal relationship between doping (cause) and a biomarker of doping (effect).

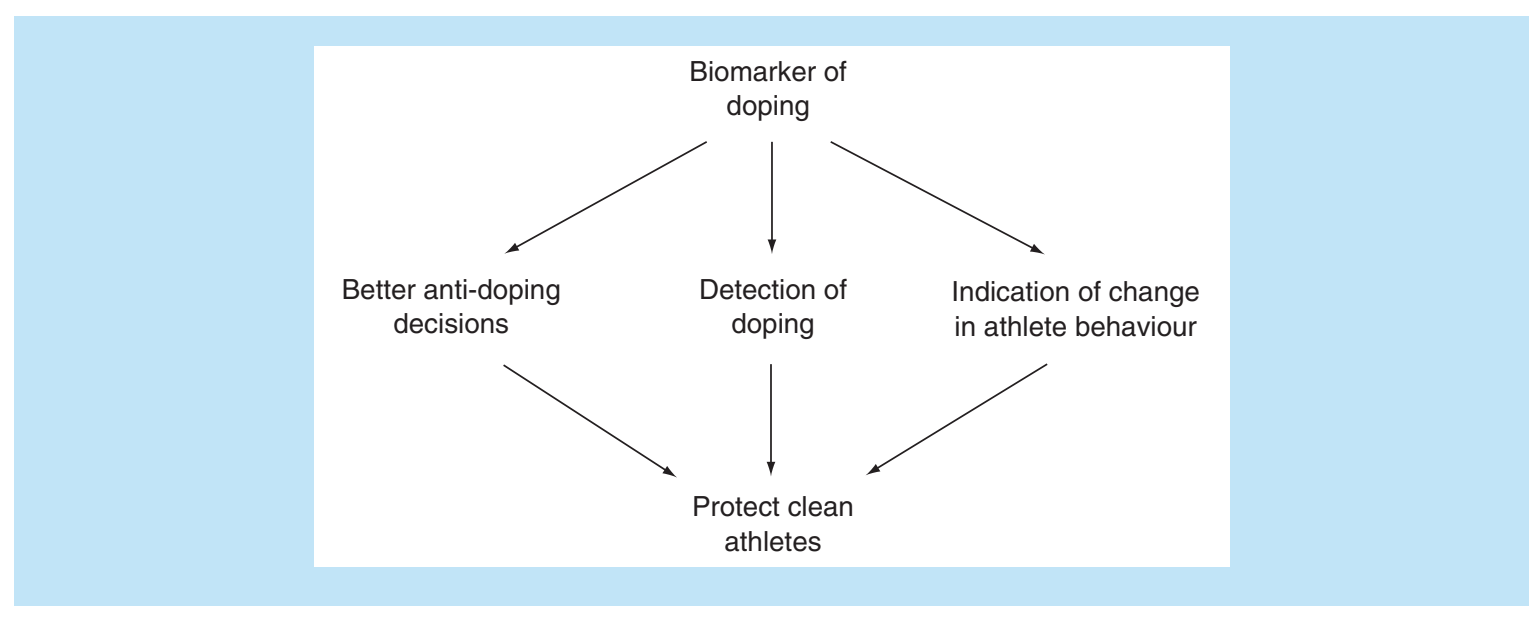

Figure 2. Use of biomarkers of doping for antidoping purposes.

\section{Table 1. Considerations for biomarker development for antidoping.}

Step Considerations

Substance choice - Prevalence and effectiveness of the substance or method

- Performance characteristics of existing markers

- Type of biomarker needed - doping, confounding factor and normalizing factor

Discovery - Substance administration (concentration, route, duration and frequency)

- Subjects - number, age and fitness level

- Matrix and frequency of sampling

- Analytics - number of targets, dynamic range, analytical uncertainty and potential for translation

\begin{tabular}{|ll}
\hline Candidate selection & - Specificity and sensitivity \\
- Low variance in baseline samples \\
- Orthogonal markers \\
- Complementary temporal response \\
- Known confounders and sources of \\
\hline Validation & - Detailed understanding of sources of \\
- Validate strategies to control confou & - Prospective trial to demonstrate imp \\
\hline Implementation & - Safety and ease of sample collection \\
& - Cost and ability to multiplex \\
- Interlaboratory analytical variability \\
- Method stability over time
\end{tabular}

considerations for strategies aimed at the discovery, validation and implementation of novel biomarkers of doping (Table 1). 


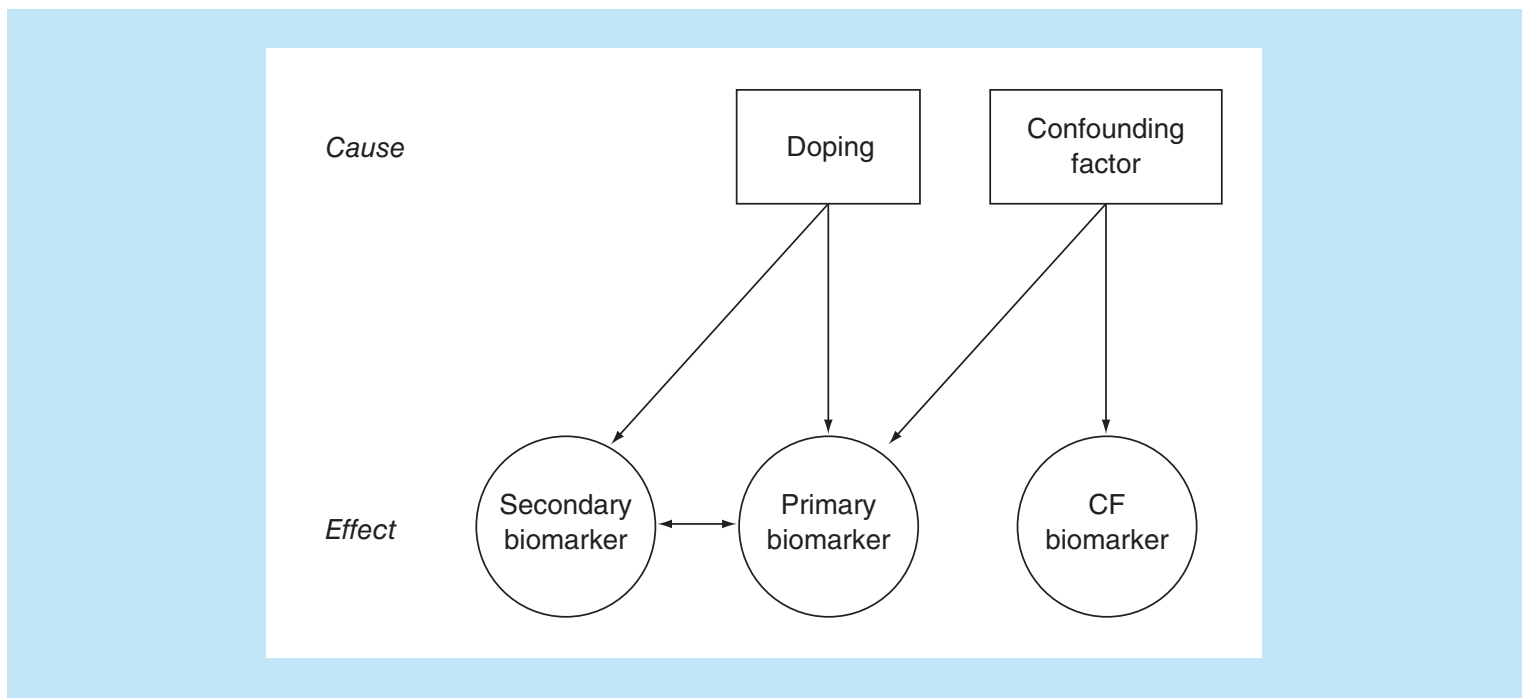

Figure 3. Different types of biomarkers of doping.

CF: Confounding factor.

\section{Biomarkers of doping}

A 'biomarker of doping' is a biological variable that indicates the response (i.e., the effect), with a high degree of certainty, to the use of a prohibited substance or method (i.e., the cause). The main biomarker performance characteristics most commonly employed in antidoping are 'sensitivity' and 'specificity', as these do not depend on knowledge of the prevalence of doping, which remains poorly characterized across elite sports. Sensitivity and specificity can be estimated at many steps during biomarker development, with the gold standard being during a prospective and controlled administration trial. Furthermore, while the ability of a biomarker of doping to identify true positives is a necessary performance characteristic of a biomarker of doping (sensitivity), the avoidance of false positives (specificity) is paramount in the context of antidoping in sport. Indeed, as the consequences of an antidoping sanction for the athlete are severe, ensuring a suitably elevated level of biomarker specificity should be a key performance target at all stages of biomarker discovery and validation.

Depending on the performance characteristics of the biomarker in question, it can serve different purposes in driving antidoping decisions and procedures. A 'primary biomarker of doping' has performance characteristics such that it can be used as primary evidence of doping. Within the context of the ABP, primary biomarkers are automatically processed by a statistical model that predicts for an individual an expected range within which a series of biomarker values falls assuming a normal physiological condition. Primary biomarker values falling outside such personalized ranges generate automatic notifications to the antidoping organization and require mandatory review by an independent expert for that particular biomarker. Examples of primary biomarkers used in antidoping include hemoglobin (HGB) concentration and the urinary testosterone to epitestosterone ratio (T/E). However, other types of biomarkers, which may not be suitable as primary biomarkers of doping, can also be valuable to antidoping (see Figure 3).

'Secondary biomarkers of doping' can provide supporting evidence to the information provided by a primary biomarker but are not sufficiently discriminant to establish doping on their own for reasons such as insufficient specificity or elevated analytical uncertainty. An example of a secondary biomarker of blood doping would be the immature reticulocyte fraction, which can provide supporting evidence of altered erythropoiesis, but with an elevated within-subject variance relative to the magnitude of variation induced by doping. Therefore, immature reticulocyte fraction has less statistical power to discriminate doping from other potential confounders.

Biomarkers of confounding factors are useful to control for potential effects of known confounding factors for a given primary biomarker. For example, the $\mathrm{T} / \mathrm{E}$ ratio is well known to be affected by the consumption of alcohol [11]. Therefore, the analysis of ethanol metabolites in urine can be used to confirm or exclude the possible confounding effects of ethanol consumption on endogenous steroid concentrations in urine [12].

Biomarkers of doping come in several forms, such as concentrations (e.g., HGB), percentages (e.g., reticulocyte percentage), ratios (e.g., T/E) or multivariate 'scores' (e.g., the 'OFF-Score' or Abnormal Blood Profile Score), each 
of which comes with differing characteristics. Concentration based markers are easier to understand conceptually and to explain to a disciplinary panel composed of nonscientists but are influenced by any factor that affects the volume of fluid in which the marker is quantified. Percentages, on the other hand, are refractory to such changes in matrix volume, such as plasma volume shifts for the blood or dilution status of the urine. Ratios can also provide a normalizing aspect which can correct for variations in concentrations with the addition of potentially increasing the dynamic range when the numerator and denominator have opposite directionality of response to doping. Such can be the case with the $\mathrm{T} / \mathrm{E}$ ratio or another ratio $(5 \alpha \mathrm{Adiol} / \mathrm{E})$ including one of the major testosterone metabolites $5 \alpha$-androstane-3 $\alpha, 17 \beta$-diol ( $5 \alpha$ Adiol) and epitestosterone [13,14]. Finally, the use of pattern classification tools can lead to more complex discriminant functions based on multiple biomarkers, such as the Abnormal Blood Profile Score [15].

Despite the successful application of some biomarkers in antidoping, there remains significant room for improvement. In particular, the window of detection of some substances is relatively short, the sensitivity of certain biomarkers still leaves a window of opportunity to dope with reduced but potentially effective doses and there are important confounding factors without viable markers or correction factors. Therefore, biomarker discovery efforts aimed at addressing these needs to have a strong potential to improve antidoping outcomes.

\section{Discovery of novel biomarkers of doping}

The field of antidoping is arguably well positioned for successful biomarker discovery compared with many disease related fields. In studies of disease, it is extremely difficult to obtain samples from the same individual in both the normal and diseased states in order to monitor biomarker response at the individual level. In antidoping trials, on the other hand, the specific response of selected biomarkers to the intervention can be followed in the same individual, allowing for collection of information on the magnitude of change relative to the baseline and interindividual differences in responses. Second, while disease progression can be variable between individuals in a given trial, even within established disease stages, the substance and dose can be tightly controlled in doping trials allowing for a very homogenous intervention and more reliable response. In addition, antidoping is also a field with previous marker discovery and implementation experience, especially with regards to metabolites, and this experience can be leveraged toward biomarker development.

Despite these potential advantages, the design of studies for the discovery of biomarkers of doping requires key considerations made in any field, starting with collecting sufficient samples from a sufficient number of individuals to provide adequate statistical power for reliable biomarker discovery. The result of underpowered discovery studies includes a decreased likelihood of detecting meaningful effects, the potential for overestimation of effect size and overall reduced reproducibility of results [16]. Thus, appropriate statistical support is essential at an early stage of study design to ensure the study is properly powered for discovery, taking into consideration the effect size sought and the number of candidate biomarkers being screened [17]. The use of robust baseline sampling and measurement of within-subject changes in biomarkers over time using a longitudinal approach, which are very feasible in antidoping biomarker discovery trials, can further improve the chances of successfully identifying reproducible biomarkers.

In terms of discovery trial design, whenever possible, human clinical trials clearly offer the best chance to uncover biomarkers relevant for human doping and avoid the discovery of biomarkers in other animal models which do not necessarily translate well into the human setting. The route of administration and the dose of the doping substance used in a discovery trial should be defined to elicit a reliable physiological response in order to promote the discovery of alterations in potentially relevant biomarkers. The sensitivity of these biomarkers to lower doses can then be specifically challenged and refined at later stages, however, the goal of the discovery phase is to uncover the highest number of relevant candidate biomarkers. Thus, while athletes may report using lower doses, the dose of the substance used for discovery should not necessarily aim to mimic these low, potentially ineffective, doses as this may reduce the ability to uncover candidate biomarkers in the first place.

As biomarkers of doping must be able to identify doping at the individual level, discovery trial design and data analysis must be designed and carried out at the individual, not population, level. In terms of study design, multiple baseline samples are required to establish the basal levels of the individual and provide valuable information on the within-subject variations. In general, 3-4 baseline samples taken over a 4-week period is a good starting point as it provides a good approximation of short-term variations due to small differences in factors such as diet, hydration status, exercise and the menstrual cycle in females. During data analysis, the ability to flag changes in biomarker patterns at the individual level must be considered, not average changes in the biomarker levels between control 
and treatment groups, where the latter may miss useful biomarkers that are masked by interindividual differences or uncover average differences that are not necessarily applicable at the individual level.

The choice of biological matrices to collect during discovery trials is also a critical consideration. The type of matrix used for antidoping purposes is tightly regulated and is based on considerations such as, accessibility at any time, the safety of the athlete, decreasing the potential for sample manipulation, stability during transport and costs related to the collection and storage of these samples. From the beginning of the fight against doping, urine has been the gold standard matrix with the advantages of a noninvasive collection procedure and an extensive window of detection for prohibited substances and their metabolites. Furthermore, since the 1970s, antidoping scientists have gained strong experience in dealing with issues related to metabolic pathways involved in the excretion of xenobiotics and doping agents. Following urine, blood samples were also introduced in order to detect blood doping and hGH misuse. Currently, both serum and EDTA-blood samples are collected for antidoping purposes. While collection of blood is often considered more invasive and transport conditions are much more challenging for antidoping organizations compared with urine, the pharmacokinetics of blood-born biomarkers benefit from the expertise of clinical medicine. More recently, alternative matrices like dried blood spots have become of interest for the antidoping community, in particular due to the ease of sample collection, transport and storage. Consequently, dried blood spots should also be considered as a matrix for biomarker discovery, acknowledging that quantification and sensitivity could be an issue due to the limited amount of biological material collected. Finally, the chemical composition and the bioavailability of putative biomarkers may also influence the choice of matrices to collect during the study protocol.

In terms of the choice of screening technology, biomarker discovery trials for antidoping can benefit from the analytical experience of antidoping laboratories. It is worth noting that currently all methods used in antidoping laboratories involve the detection or quantification of substances, metabolites or proteins. In particular, antidoping laboratories have strong expertise in mass spectrometry (MS), where validated MS-based methods have withstood legal challenge. MS also offers the ability to multiplex different biomarkers during routine antidoping sample analysis; so MS-based screening approaches offer the potential for more rapid and cost-effective translation into routine practice. Additionally, many antidoping laboratories have the capacity to carry out high-resolution metabolomic analyses in house, where knowledge of routine sample preparation methods can inform analytical considerations during this discovery phase, which may further improve the potential for translation of candidate biomarkers into routine use. Other screening tools not based on MS can be considered, such as affinity-based screening tools, where the eventual choice of analytical approach upon implementation could still be MS, where possible. Finally, screening for alterations in RNA levels has shown potential to identify transcriptional biomarkers for discriminating doped individuals $[18,19]$, though quantification of RNA remains to be validated as an analytical approach for antidoping purposes.

Particular discovery method performance characteristics that should be considered at this stage are analytical uncertainty, dynamic range and to a lesser extent, the number of targets measured by the method in question. Considering that target validation will address issues of specificity, the goal is to ensure the most favorable signalto-noise ratio for the highest number of targets.

\section{Considerations for candidate selection for further validation}

The strategy for data analysis and candidate selection should be designed to take advantage of the opportunities afforded by the control over the discovery trial design, which include the longitudinal nature of the data and the baseline information for each individual. Among selection criteria to consider during this phase, the performance characteristics of the biomarkers (sensitivity and specificity) are critical, with particular importance placed on specificity. Additionally, the ratio of the within/between-subject variance (also known as the index of individuality' [20]) can be estimated from the baseline and control samples of the clinical trial, where a lower ratio can be favored when prioritizing candidates for further validation for use in the ABP. Similarly, models comparing the treatment-induced variance relative to the within-subject variance can provide an indication of the discriminatory potential of candidate biomarkers to detect doping beyond normal individual variation. The use of mixed model approaches can be particularly useful for longitudinal, multi-dimensional biological datasets [21,22].

It is also valuable to consider whether the candidate biomarker is orthogonal to existing biomarkers in terms of its known biological function [23]. In other words, is the candidate biomarker involved in distinct biological functions/pathways compared with existing biomarkers or is it closely related to existing biomarkers? The use of orthogonal biomarkers arguably increases the chances of having a more specific signature of a response to doping 
and also may decrease the likelihood that all biomarkers would be equally affected by a given confounding factor. Unlike hypothesis-driven candidate selection, the ability to uncover orthogonal biomarkers is a clear advantage of applying unbiased screening approaches during the discovery phase.

Higher priority should also be given to candidates that show a complementary temporal response to doping, which may extend the window of detection. These could either be biomarkers that respond more rapidly or more slowly than existing biomarkers. It may also be interesting to consider biomarkers that have different responses in terms of directionality of response (e.g., upregulated vs downregulated), where the use of a ratio of the two may provide improved sensitivity for the detection of doping. Similarly, combining several biomarkers into 'scores' or 'indices' can improve performance characteristics compared with any one biomarker alone. Finally, a literature search can reveal other potential caveats such as diurnal variations, known confounding factors, genomic variants, or disease-related and/or age-related changes that can be used to prioritize candidate biomarkers.

\section{Biomarker validation}

Once a prioritized shortlist of candidate biomarkers is made, a more rigorous determination of the sources of variation must be carried out in conjunction with a prospective validation of the ability of the biomarker to improve antidoping outcomes.

An explicit understanding of the sources of variation of a given biomarker is critical to establishing its usefulness in antidoping and for its implementation into routine practice. The main sources of biomarker variation can be grouped into those due to variations between individuals (between subject) and variations within individuals (within-subject), the latter of which includes biological variation, variations due to sample collection/transportation and analytical variations. While some preliminary variance estimates can be made during the discovery phase, a more rigorous determination of the sources of variance must be carried out on a larger population over a longer period. Elite athletes have the choice to allow their real antidoping samples to be used for research following analysis for antidoping purposes. Such samples are ideal for studying biomarker variation in true elite athlete samples, with the caveat that such samples may include those from doped athletes.

Between-subject variation often contributes to a significant portion of the total variation of biomarker. For example, HGB has a between-subject variance that is approximately twice as much as the within-subject variance. Therefore, the passport approach, which removed much of the between-subject variance, leads to improved sensitivity compared with using population-based values for HGB. These between-subject differences can be due to factors such as ethnicity, gender, environmental conditions or diet. Within-subject variability in biomarkers can be due to factors such as, diurnal variation, diet, exercise, training status (e.g., in-season vs off-season), medications or specific medical conditions. Biomarkers that change with age can affect both within- and between-subject variations.

Knowledge of previous legal challenges of existing biomarkers and proposed confounding factors in specific cases can provide a scale of the importance of carrying out a thorough examination of all plausible confounders [24,25]. Knowing that the eventual likelihood ratio to be evaluated when advancing a sanction will be weighting the likelihood of doping against the likelihood of all other causes, researchers should come up with an exhaustive list of plausible hypotheses of causes of biomarker variation, other than doping, and evaluate how well these are understood and how they could eventually be controlled. Such propositions can be grouped broadly into preanalytical factors, analytical issues, environmental factors and pathologies [24].

Once the sources of variation and confounding factors of a biomarker are well characterized, different strategies can be considered in order to control them within antidoping procedures. These can be controlled at the preanalytical level, the analytical level, within the statistical calculations in the ABP or as information given to the experts reviewing the athlete's passport. It is important to note that sample collection, transportation and analysis can be highly harmonized in antidoping. Thus, analytical or preanalytical issues which are uncovered during the discovery and/or validation phases may be addressed through specific protocols. For example, it is known that there are differences in the measurement of reticulocyte percentage on different blood cell analyzers [26]. For this reason, all antidoping laboratories are equipped with blood analyzers based on similar technology. Similarly, postural changes are known to affect plasma volume; thus athletes must be seated for 10 min prior to blood collection for the ABP [27]. Heterogeneous factors that can be readily modeled can be incorporated directly into the calculation of the population-based limits of the ABP, such as gender-based differences in mean HGB levels [28]. Information on factors that are more difficult to model, for example the effect of altitude on blood parameters, can simply be provided as raw data for the experts to evaluate in the context of their review of the passport. The degree to which a 
specific procedure can control such issues can be specifically tested during biomarker validation and will ultimately decide whether to continue development of such a biomarker or not.

While theoretically not essential for a biomarker with strong performance characteristics to indicate doping, an understanding of the underlying biological response of a biomarker to doping can help justify its use during legal proceedings. In particular, the ability to explain to a hearing panel why this particular biomarker is responding to doping can help to give more weight to the evidence it provides, allowing the panel to confidently use it to inform their assessment of the likelihood of doping.

Finally, the gold standard for candidate doping biomarker validation remains the testing of the performance of the biomarker, including proposed decision criteria, in independent prospective clinical trials. Where available, such validation trials should include comparison with existing biomarkers in order to demonstrate an improvement that would justify the implementation of a new biomarker. For obvious reasons, such administration trials cannot be performed on competing elite athletes, however, a well-designed trial using recreational athletes is sufficient to allow validation of the biomarker response. Such trials should seek to mimic as closely as possible real antidoping sampling rates. In practice, it is unlikely that an antidoping organization collects samples at the same frequency as the sampling rates used during clinical trials. Thus, it is expected that more realistic sampling rates will reduce the sensitivity of the considered biomarkers. A second validation phase with a random selection of samples from each period should be carried out to take a certain degree of imperfect observation into account (see for example [29]). Ideally, even the order (and the number) of periods from which the samples are drawn should be randomly selected to mimic the observed stochastic nature of real-word antidoping data, where, for example, the first sample in the sequence may be affected by doping. Only biomarkers which demonstrate a sufficient improvement in antidoping outcomes should then be considered for implementation.

\section{Implementation}

Once a biomarker has been shown to have a positive impact on antidoping outcomes, consideration can be made for implementation of a method for the reliable measurement of this new biomarker. Factors such as safety of the method, ease of collection of the sample, cost and inter-laboratory analytical variability will be key determinants of the decision to implement the new biomarker.

A cost-effective analytical strategy is required for each biomarker of doping which can be harmonized across multiple labs in order to produce comparable results regardless of where the samples may be analyzed. The ability to multiplex several biomarkers into one assay is clearly advantageous in order to reduce costs while biomarkers requiring dedicated laboratory equipment and reagents are less favorable.

The stability of the detection method over time is also a key consideration, especially when a longitudinal approach is used. As equipment or reagent changes can affect biomarker values, it is important to consider that affinity-based detection methods are subject to potential changes in reagents over time. The current trend in antidoping is to focus on chromatographic methods coupled to MS, which allows the inclusion of multiple compounds and biomarkers with high selectivity and sensitivity. Most analyses are currently performed using a targeted approach knowing the identity of the compounds to detect from the World Anti-Doping Agency Prohibited List. Nevertheless, the quantification of proteins by chromatographic methods coupled to MS remains difficult, with generally poorer precision compared with affinity-based methods. Thus, the continued improvement of MS-based quantification methods for proteins is expected to directly impact the successful application of protein biomarkers in antidoping.

\section{Future perspective}

While traditionally antidoping has focused on substance detection and identification, there is a clear emphasis on biomarker development as a complementary approach to uncover doping. As the ABP provides a legally validated framework for the use of biomarkers, a key goal of antidoping research is the continuous development of biomarkers of doping for implementation within this framework.

There are currently several ongoing efforts, each at different stages of development, aiming to integrate new biomarkers in the ABP. While the current hGH biomarker assay is built on population-based thresholds calculated using two protein biomarkers (IGF-I and P-III-NP) [30], developments are underway to apply this approach in a longitudinal manner in the ABP. The recent development of biomarkers of plasma volume, a key confounding factor for the hematological module of the ABP, has demonstrated the ability of pragmatic biomarker discovery trial design to uncover useful biomarkers with a potential for translation into routine use [31-33]. Recognizing the limitations of current markers of the hematological module of the ABP, the World Anti-Doping Agency recently 
launched targeted funding for discovery of novel biomarkers of blood doping and altitude exposure [34]. Discovery trials for new markers of hGH doping and steroid doping are also ongoing [35].

Therefore, biomarker development efforts in antidoping are producing viable candidates that are progressing toward validation and possible future implementation and it is expected that this trend will continue to accelerate as we see continued investment in biomarker research, continuously improving analytical tools and increased experience with biomarker development in antidoping.

\section{Conclusion}

Biomarker discovery and development remains very difficult across all fields, however, significant progress is being made at several steps, including analytics, data analysis and discovery trial design. Antidoping remains a small field compared with other biomarker discovery efforts and collaborations with biomarker discovery experts from other fields will clearly be beneficial. With access to elite athlete samples, a harmonized network of laboratories and a

\section{Executive summary}

Biomarkers of doping

- A 'biomarker of doping' is a biological variable that indicates the biological response to the use of a prohibited substance or method.

- The use of biomarkers in antidoping has proven beneficial not only to directly sanction athletes, but also to drive many other antidoping decisions.

- The Athlete Biological Passport (ABP) provides a harmonized, legally validated framework for the use of biomarkers in antidoping.

- A key target of antidoping science is the continued improvement of the ABP through the development of novel biomarkers of doping.

Discovery of novel biomarkers of doping

- The field of antidoping is arguably well positioned for successful biomarker development because of: the ability to carry out controlled longitudinal administration studies; access to elite athlete samples for research; a harmonized network of antidoping laboratories and analytical tools.

- The success of biomarker discovery trials in antidoping depends on key considerations such as favoring human trials where possible, ensure adequate study power including robust baseline sampling, choosing analytical approaches with favorable signal-to-noise ratios and carrying out data analysis with the aim to discriminate doping at the individual level.

Considerations for candidate selection for further validation

- When choosing candidates for further validation, the performance characteristics of the biomarkers (sensitivity and specificity) are critical, with particular importance placed on specificity in antidoping and controlling the possibility of false positives.

- Other factors to consider are the within- and between-subject variance, whether the candidate biomarker is functionally orthogonal to other existing biomarkers and information on potential confounders such as, diurnal variations, genomic variants, disease-related and/or age related changes.

Biomarker validation

- In order to validate a candidate biomarker for routine use, a more rigorous determination of the sources of variation must be carried out in conjunction with a prospective validation of the ability of the biomarker to improve antidoping outcomes.

- The gold standard for candidate biomarker validation remains the testing of the performance of the biomarker, including proposed decision criteria, in independent prospective clinical trials.

Implementation

- Once a biomarker has been shown to improve antidoping outcomes, consideration can be made for implementation of a method for the reliable measurement of this new biomarker.

- Factors such as, safety of the method, ease of collection of the sample, cost and inter-laboratory analytical variability will be key determinants of the decision to implement the new biomarker.

Future perspective

- While traditionally antidoping has focused on substance detection and identification, there is a clear emphasis on continued biomarker development as a complementary approach to uncover doping.

- Biomarker development efforts in antidoping are producing viable candidates that are progressing toward implementation and it is expected that this trend will continue to accelerate as we see continued investment in biomarker research, improving analytical tools and increased experience with biomarker development in antidoping. 
legally validated framework already in place for the use of biomarkers, antidoping is well positioned for improving biomarker validation and implementation.

Financial \& competing interests disclosure

The authors have no relevant affiliations or financial involvement with any organization or entity with a financial interest in or financial conflict with the subject matter or materials discussed in the manuscript. This includes employment, consultancies, honoraria, stock ownership or options, expert testimony, grants or patents received or pending, or royalties.

No writing assistance was utilized in the production of this manuscript.

\section{Open access}

This work is licensed under the Attribution-NonCommercial-NoDerivatives 4.0 Unported License. To view a copy of this license, visit http://creativecommons.org/licenses/by-nc-nd/4.0/

\section{References}

Papers of special note have been highlighted as: $\bullet$ of interest; $\bullet \bullet$ of considerable interest

1. Sottas PE, Robinson N, Rabin O, Saugy M. The athlete biological passport. Clin. Chem. 57(7), 969-976 (2011).

2. Sottas PE, Robinson N, Fischetto G, Dollé G, Alonso JM, Saugy M. Prevalence of blood doping in samples collected from elite track and field athletes. Clin. Chem. 57(5), 762-769 (2011).

3. Zorzoli M. Biological passport parameters. J. Hum. Sport Exerc. 6(2), 205-217 (2011).

4. World Anti-Doping Agency. WADA Independent Commission Report 1, Chapter 11 (2015). http://www.wada-ama.org/sites/default/files/resources/files/wada_independent_commission_report_1_en.pdf

5. Cycling Independent Reform Commission. Report to the President of the Union Cycliste Nationale (2015). http://www.uci.org/docs/default-source/imported-library/circreport2015_neutral.pdf?sfvrsn=7a9764d3_14

6. Rifai N, Gillette MA, Carr SA. Protein biomarker discovery and validation: the long and uncertain path to clinical utility. Nat. Biotechnol. 24(8), 971-983 (2006).

7. Ioannidis JPA, Bossuyt PMM. Waste, leaks, and failures in the biomarker pipeline. Clin. Chem. 63(5), 963-972 (2017).

8. McDermott JE, Wang J, Mitchell $\mathrm{H}$ et al. Challenges in biomarker discovery: combining expert insights with statistical analysis of complex omics data. Expert Opin. Med. Diagn. 7(1), 37-51 (2013).

9. Ignjatovic V, Geyer PE, Palaniappan KK et al. MS-based plasma proteomics: considerations from sample collection to achieving translational data. J. Proteome Res. 18(12), 4085-4097 (2019).

10. Wishart DS. Emerging applications of metabolomics in drug discovery and precision medicine. Nat. Rev. Drug Discov. 15(7), 473-484 (2016).

11. Falk O, Palonek E, Björkhem I. Effect of ethanol on the ratio between testosterone and epitestosterone in urine. Clin. Chem. 34(7), 1462-1464 (1988).

12. Grosse J, Anielski P, Sachs H, Thieme D. Ethylglucuronide as a potential marker for alcohol-induced elevation of urinary testosterone/epitestosterone ratios. Drug Test. Anal. 1(11-12), 526-530 (2009).

13. Ayotte C. Detecting the administration of endogenous anabolic androgenic steroids. Handb. Exp. Pharmacol. 195, 77-98 (2010).

14. Geyer H, Flenker U, Mareck U et al. The detection of the misuse of testosterone gel. In: Proceedings of the 15th Cologne Workshop on Dope Analysis SPORT undBUCH Strauss. Schänzer W, Geyer H, Gotzmann A, Mareck U (Eds). Köln, Germany, 123-142 (2007).

15. Sottas PE, Robinson N, Giraud S et al. Statistical classification of abnormal blood profiles in athletes. Int. J. Biostat. 2(1), 1557-4679 (2006).

16. Button KS, Ioannidis JP, Mokrysz C et al. Power failure: why small sample size undermines the reliability of neuroscience. Nat. Rev. Neurosci. 14(5), 365-376 (2013).

17. Krzywinski M, Altman N. Power and sample size. Nat. Methods. 10, 1139-1140 (2013).

18. Durussel J, Haile DW, Mooses K et al. Blood transcriptional signature of recombinant human erythropoietin administration and implications for antidoping strategies. Physiol. Genomics 48(3), 202-209 (2016).

19. Wang G, Durussel J, Shurlock J et al. Validation of whole-blood transcriptome signature during microdose recombinant human erythropoietin (rHuEpo) administration. BMC Genomics 18(Suppl. 8), 817 (2017).

20. Harris EK. Effects of intra- and interindividual variation on the appropriate use of normal ranges. Clin. Chem. 20, 1535-1542 (1974).

21. Gibbons RD, Hedeker D, DuToit S. Advances in analysis of longitudinal data. Annu. Rev. Clin. Psychol. 6, 79-107 (2010).

22. Bates D, Machler M, Bolker B, Walker S. Fitting linear mixed-effects models using lme4. J. Stat. Softw. 67, 1-48 (2015).

23. Gerszten RE, Wang TJ. The search for new cardiovascular biomarkers. Nature 451 (7181), 949-952 (2008). 
24. Schumacher YO, d'Onofrio G. Scientific expertise and the Athlete Biological Passport: 3 years of experience. Clin. Chem. 58(6), 979-985 (2012).

-• Outlines key lessons from the first Athlete Biological Passport case hearings and appeals to the Court of Arbitration for sport.

25. Kuuranne T, Saugy M, Baume N. Confounding factors and genetic polymorphism in the evaluation of individual steroid profiling. Br. J. Sports Med. 48(10), 848-855 (2014).

26. Ashenden MJ, Sharpe K, Damsgaard R, Jarvis L. Standardization of reticulocyte values in an antidoping context. Am. J. Clin. Pathol. 121(6), 816-825 (2004).

27. Ahlgrim C, Pottgiesser T, Robinson N, Sottas PE, Ruecker G, Schumacher YO. Are 10 min of seating enough to guarantee stable haemoglobin and haematocrit readings for the athlete's biological passport? Int. J. Lab. Hematol. 32(5), 506-511 (2010).

28. Sottas PE, Robinson N, Saugy M, Niggli O. A forensic approach to the interpretation of blood doping markers. LawProbab.Risk 7(3), 191-210 (2008).

-• Describes the original assumptions and statistical approaches underlying the passport approach for the hematological module.

29. Pottgiesser T, Echteler T, Sottas PE, Umhau M, Schumacher YO. Hemoglobin mass and biological passport for the detection of autologous blood doping. Med. Sci. Sports Exerc. 44(5), 835-843 (2012).

30. Holt RI, Böhning W, Guha N et al. The development of decision limits for the GH-2000 detection methodology using additional insulin-like growth factor-I and amino-terminal pro-peptide of type III collagen assays. Drug Test. Anal. 7(9), 745-755 (2015).

31. Lobigs LM, Sottas PE, Bourdon PC et al. The use of biomarkers to describe plasma-, red cell-, and blood volume from a simple blood test. Am. J. Hematol. 92(1), 62-67 (2017).

- Describes a pragmatic approach to screening for markers of plasma volume with a high potential for translation.

32. Lobigs LM, Sottas PE, Bourdon PC et al. A step towards removing plasma volume variance from the Athlete's Biological Passport: the use of biomarkers to describe vascular volumes from a simple blood test. Drug Test. Anal. 10(2), 294-300 (2018).

33. Lobigs LM, Garvican-Lewis LA, Vuong VL et al. Validation of a blood marker for plasma volume in endurance athletes during a live-high train-low altitude training camp. Drug Test. Anal. 10(7), 1176-1183 (2018).

34. World Anti-Doping Agency. Request for Applications - Targeted Research - Markers of Erythropoiesis Stimulating Agents (ESA) use and Hypoxia (2020). http://www.wada-ama.org/sites/default/files/resources/files/rfa_-_targeted_research_-_esa_hypoxia_english-final_0.pdf

35. World Anti-Doping Agency. Funded Research Projects (2020).

https://www.wada-ama.org/en/what-we-do/science-medical/research/funded-research-projects 